\title{
Takayasu arteritis presenting as renovascular hypertension with hypertensive encephalopathy
}

\author{
Vikram Singh Tanwar, ${ }^{1 *}$ Anjali Saini, ${ }^{2}$ Anurag Ambroz Singh, ${ }^{1}$ Rakesh Tank, ${ }^{1}$ \\ ${ }^{1}$ Department of medicine, SHKM GMC Nalhar (122107) India, ${ }^{2}$ PGIMS Rohtak (124001) India
}

\begin{abstract}
DOI Name
http://dx.doi.org/10.3126/jaim.v6i2.18540

Keywords

Takayasu arteritis, vasculitis, hypertensive

encephalopathy

Citation

Vikram Singh Tanwar, Anjali Saini, Anurag Ambroz Singh, Rakesh Tank. Takayasu arteritis presenting as renovascular hypertension with hypertensive encephalopathy. Journal of Advances in Internal Medicine 2017;06(02):35-37.
\end{abstract}

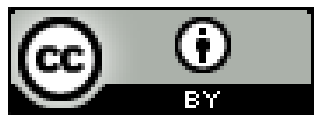

This work is licensed under a Creative Commons Attribution 3.0 Unported License.

\begin{abstract}
Takayasu arteritis is a large vessel vasculitis that has variable presentation. It is suspected when there are pulse and BP discrepancies between upper limbs or absent pulses. We here presenting a case of takayasu arteritis that remained undiagnosed till 40 years and at first time manifested with hypertensive encephalopathy and managed well with medical therapy.
\end{abstract}

\section{INTRODUCTION}

Takayasu Arteritis (TA) is a type of chronic granulomatous vasculitis of unknown cause (majority of authors agree on its autoimmue etiology). It has worldwide distribution, with the greatest prevalence among Asians. TA commonly manifest with various constitutional symptoms like fever, bodyache, weakness but sometimes it remains undiagnosed till late and manifest primarily with uncommon presentations like hypertensive encephalopathy. This report is representing such an uncommon presentation of a rare disease.

\section{CASE REPORT}

A 42 years old lady presented in the emergency department with complaints of headache, dizziness, confusion, and blurring of vision for 5 days. There was history of nonspecific complaints like bodyache, generalized body weakness, fatigue, low energy and difficulty in doing daily routine activities for last 6 months. There was no history of loss of consciousness/ abnormal body movements/ joint pain/weight loss/fever/ anorexia/ cough with or without expectoration. Patient had never been detected as having high blood pressure before this.

On examination patient was found to have high blood pressure i.e. $210 / 130 \mathrm{mmHg}$ (measured in right arm taken in supine position) but in left arm BP was not recordable. Pulse (radial and brachial) was not palpable in left upper limb. Pulse was well palpable in right upper limbs and both lower limbs. No other abnormal clinical finding was detected on cardiovascular, respiratory and neurological examination.

In the emergency, first high BP was managed with antihypertensive agents which lowered down to $150 / 90 \mathrm{mmHg}$ and then MRI brain was done that showed slight parenchymal enhancement in the occipital areas and both cerebral hemispheres suggestive of hypertensive changes. Patient was admitted with provisional diagnosis of hypertensive encephalopathy and further workup was planned to find out the secondary cause of hypertension.

As patient presented with BP and pulse discrepancies in both upper limbs in the background of severe hypertension, so we initially thought that patient might have large vessel vasculitis possibly involving branches of aortic arch and renal arteries.

\footnotetext{
* Corresponding author

Vikram Singh Tanwar, Assistant professor

Department of Medicine

SHKM GMC Nalhar (122107) Nuh, India

Email address-drvikrampgi@gmail.com
} 
So we went for CT angiography (CTA) for both thoracic aorta and abdominal aorta and their branches. CTA thoracic aorta revealed the multiple areas of intimal thickening of aortic arch and its branches; irregularity with stenosis at the origin of left common carotid arteries, and occlusion of the left subclavian artery. CTA of abdominal aorta and its branches revealed right renal artery stenosis, diffuse intimal thickening with dilation and narrowing of abdominal aorta.

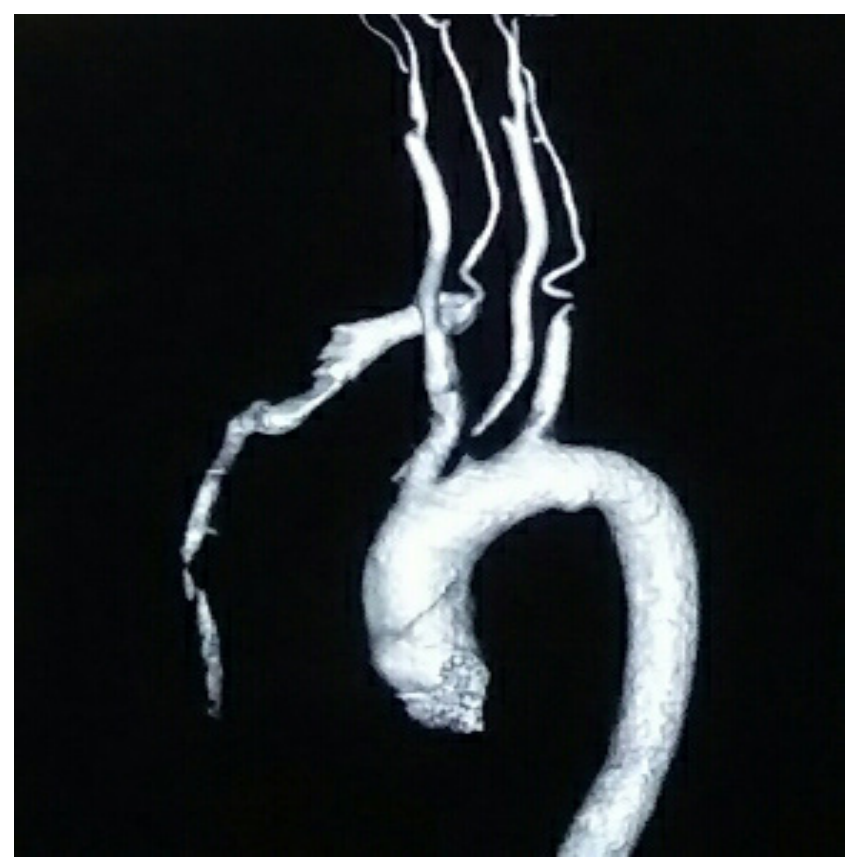

Figure 1: CTA of thoracic aorta revealing the multiple areas of intimal thickening of aortic arch and its branches; irregularity with stenosis at the origin of left common carotid arteries, and occlusion of the left subclavian artery

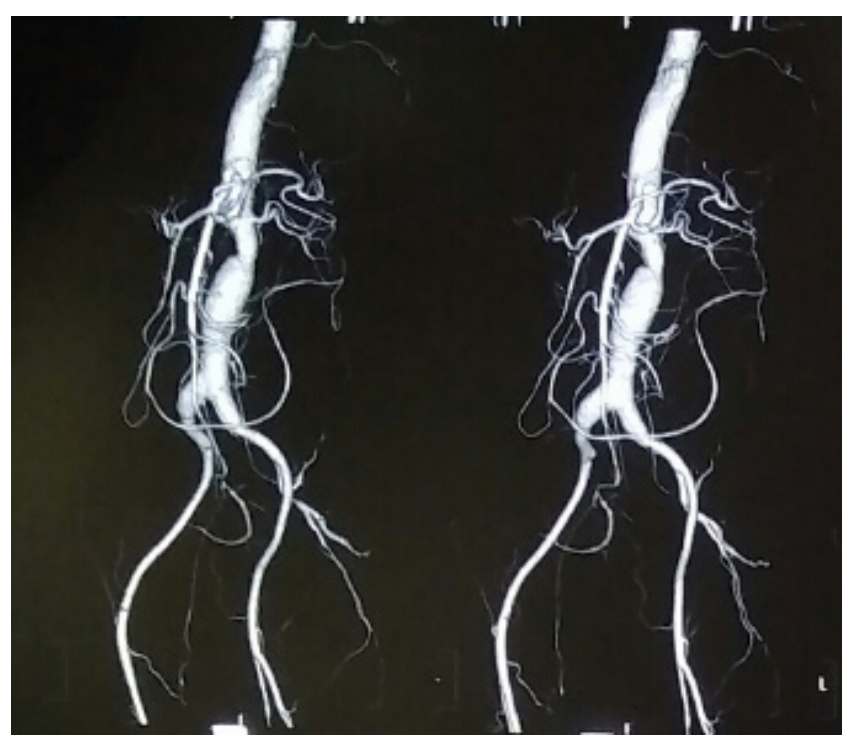

Figure 2: CTA of abdominal aorta and its branches revealed right renal artery stenosis, diffuse intimal thickening with dilation and narrowing of abdominal aorta.
The CTA reports were suggestive of Takayasu arteritis with involvement of thoracic and abdominal aorta i.e. Takayasu arteritis type V. All biochemical and hematological reports were within normal limits except CRP and ESR. Both CRP and ESR values were highly elevated. Fundus examination showed grade 4-hypertensive retinopathy in both eyes. So the final diagnosis was made as Takayasu arteritis type $V$ with hypertensive encephalopathy. We started amlodipine and Telmisartan for the hypertension and prednisolone (1mg per $\mathrm{Kg}$ body weight daily), methotrexate (15-20mg weekly) for the vasculitis. With the treatment patient responded well, headache and vision was improved, acute phase reactants came down within their normal range and patient started feeling better in term of ability to do routine activities.

\section{DISCUSSION}

TA is a rare, idiopathic, chronic inflammatory disease, characterized by granulomatous panarteritis of aorta and its major branches, typically presenting before the age of 40 years [1,2]. As far as its etiology is concern, TA is an immune mediated inflammatory process leading to the wall thickening, fibrosis, stenosis and thrombus formation. As like the other autoimmune diseases it affects the females 8 times more frequently than the males. The main arteries involved in TA are subclavian A (93\%), common carotid artery (58\%), abdominal aorta $(47 \%)$, renal artery (38\%), aortic arch or root (35\%) and vertebral A (35\%) [1]. According to New angiographic classification system, TA is classified into five types that allows a comparison of patient characteristics according to the vessels involved and is helpful in planning surgery, but offers little by way of prognosis [3].

The presentation of TA is heterogeneous. Approximately $10 \%$ of patients with TA are asymptomatic, and the diagnosis is suggested only by abnormal vascular findings on physical examination. Constitutional symptoms may precede clinical vascular involvement. Common constitutional symptoms include headache (50-70\%), malaise (35-65\%), arthralgia (2875\%), and fever (9-35\%). Neurological manifestations are headache $(50-70 \%)$, visual disturbance (16-36\%), stroke (5$9 \%)$, seizures $(0-10 \%)$. Common cardiovascular presentations are absent pulses (96\%), bruits (45-70\%), Claudication (38$81 \%)$, heart failure (7\%), and hypertension (28-50\%) $[2,4,5]$. However the cause of hypertension in TA is multifactorial, these are related to changes of vascular compliance, increased sensitivity to carotids, increased afterload due to acquired coarctation of any part of aorta, renal artery stenosis leading to vascular ischemia with hyper-reninemia. 
Early initiation of immunosuppressive treatment is crucial to control active inflammation and minimize arterial injury. Immunosuppressive agents like corticosteroids (prednisolone $1 \mathrm{mg}$ per kg body weight per day) and/or methotrexate (15$25 \mathrm{mg}$ weekly) are easily available and affordable to patients in developing countries, hence commonly used.Experience with cyclophosphamide and mycophenolate mofetil is limited [6]. There are various studies done that support the use of biologics (anti-TNF $\alpha$ ) in the treatment of TA but concern is about their high cost and availability [7].

Some patients of TA who do not respond to medical therapy or has severe stenotic lesions, need surgical intervention. Whenever possible, surgery should be delayed until clinical remission is achieved [8]. The indications for considering surgical intervention include uncontrolled hypertension due to renal artery stenosis, severe symptomatic coronary artery or cerebrovascular disease, severe aortic regurgitation, stenotic or occlusive lesions resulting in critical limb ischemia, and aneurysms at risk of rupture $[9,10]$.

In our patient aortic arch with its branches, abdominal aorta, renal artery and iliac arteries are involved which put her into type 5 TA. There was extensive involvement of medium to large

\section{REFERENCES}

1. Langford CA, Fauci AS. The vasculitis syndromes. In: Longo DL, Fauci AS, Kasper DL, Hauser SL, Jameson JL, Loscalzo J, editors. Harrison's Principles Internal medicine $19^{\text {th }}$ edi new york: Mcgraw hill; 2015. p 217993.

2. Kerr GS, Hallahan CW, Giordano J, Leavitt RY, Fauci AS, Rottem M, et al. Takayasu arteritis. Ann Intern Med. 1994 Jun 1. 120(11):919-29.

3. Moriwaki R, Noda M, Yajima M, Sharma BK, Numano F. Clinical manifestations of Takayasu arteritis in India and Japan-new classification of angiographic findings. Angiology. 1997;48:369-79.

4. Soto ME, Espinola N, Flores-Suarez LF, Reyes PA. Takayasu arteritis: clinical features in 110 Mexican Mestizo patients and cardiovascular impact on survival and prognosis. ClinExpRheumatol. 2008 May-Jun. 26(3 Suppl 49):S9-15.

5. Park MC, Lee SW, Park YB, Chung NS, Lee SK. Clinical characteristics and outcomes of Takayasu's arteritis: analysis of 108 patients using standardized criteria for diagnosis, activity assessment, and angiographic vessels along with elevated acute phase reactant suggesting very high disease activity so we started medical therapy in term of prednisolone and methotrexate in combination with antihypertensive agents and the response comes out to be very good. Her symptoms were improved; BP was controlled and absent pulses was reappeared after one month of therapy.

\section{CONCLUSION}

Primarily the report shows the uncommon presentation of a rare disease like takayasu arteritis. Secondly, in the presence of new onset hypertension or hypertensive emergency with pulse and BP discrepancies one should always suspect any vasculitic condition. In the presence of active ongoing inflammatory process the timely started immunosuppressive therapy has very good result and better prognosis.

\section{CONFLICT OF INTEREST}

The authors declare that there is no conflict of interest regarding the publication of this paper.

classification. Scand J Rheumatol. 2005 Jul-Aug. 34(4):284-92.

6. Hoffman GS, Leavitt RY, Kerr GS, Rottem M, Sneller MC, Fauci AS. Treatment of glucocorticoid-resistant or relapsing Takayasu arteritis with methotrexate. Arthritis Rheum. 1994;37:578-582.

7. Comarmond C, Plaisier E, Dahan K et al. Anti TNF- $\alpha$ in refractory Takayasu's arteritis: cases series and review of the literature. Autoimmunity Reviews. 2012;11(9):67884.

8. Min PK, Park S, Jung Jh, Ko YG, Choi D, Jung $Y$ et al. Endovascular Therapy Combined with Immunosuppressive Treatment for Occlusive Arterial Disease in Patients with Takayasu's Arteritis. J EndovascTher 2005;12:28-34.

9. Fields CE, Bower TC, Cooper LT et al. Takayasu's arteritis: operative results and influence of disease activity. Journal of Vascular Surgery. 2006;43:64-71.

10. Miyata T, Sato O, Koyama H, Shigematsu H, Tada Y. Longterm survival after surgical treatment of patients with Takayasu's arteritis. Circulation 2003;108(12):1474-80. 